\title{
Aerosol derived airway morphometry in healthy subjects
}

\author{
P. Brand, C. Rieger, T. Beinert, J. Heyder
}

Aerosol derived airway morphometry in healthy subjects. P. Brand, C. Rieger, T. Beinert, J. Heyder. C ERS Journals Ltd 1995.

ABSTRACT: Monodisperse aerosol particles can be used to non-invasively probe intrapulmonary airspace dimensions. In this study, the aerosol-derived airway morphometry technique was used to study airspace dimensions in $\mathbf{7 9}$ healthy subjects, in order to assess reference data for the future clinical application of aerosolderived airway morphometry, and to investigate the effect of lung inflation, anthropometric, and lung function parameters on aerosol-derived airway morphometry.

Intrapulmonary airspace dimensions were assessed by measuring the deposition of monodisperse, hydrophobic submicron aerosol particles during breathholding. Additionally, measurements of spirometric and body plethysmographic lung function were performed.

Airspace dimensions were in good agreement with morphometric lung data. Airspace dimensions increased with increasing lung inflation. Interindividual variation of airspace dimensions was lowest in the lung periphery, at high levels of lung inflation, and when the volumetric lung depth was normalized to the endinspiratory lung volume. Analysis of variance showed an increase of airspace dimensions with age.

The results of this study indicate that aerosol-derived airway morphometry is dependent on the level of lung inflation and the age of the subject. These results suggest that in contrast to conventional lung function techniques, aerosol-derived airway morphometry might be a powerful tool for the detection of small changes in peripheral airway geometry.

Eur Respir J., 1995, 8, 1639-1646.
GSF-Forschungszentrum für Umwelt und Gesundheit, Institut für Inhalationsbiologie, Oberschleißheim, Germany.

Correspondence: P. Brand

GSF - Forschungszentrum für Umwelt und Gesundheit, Institut für Inhalationsbiologie

Robert-Koch-Allee 6

D-82131 Gauting

Germany

\section{Keywords:}

Aerosol-derived airway morphometry ageing lung

healthy subjects

intersubject variability

lung inflation

lung models

Received: December 191994

Accepted after revision June 41995
Monodisperse aerosol particles can be used to noninvasively probe intrapulmonary airspace dimensions. The validity of airspace dimensions assessed by this technique was studied in excised human lungs [1], dog lungs $[2,3]$, and physical models [4]. This technique has been used to detect structural changes in lungs of patients with coal-miners' pneumoconiosis [5], and patients with chronic obstructive pulmonary disease (COPD) [6-8]. However, the experimental conditions differ considerably from study to study. In particular, the breathing manoeuvre used for the measurement of airspace dimensions has varied and is assumed to be responsible for some contradictory results. If the entire scope of diagnostic capabilities of this technique is to be utilized, efforts should be made to standardize the experimental conditions.

In this study, the single-breath concentration technique $[9,10]$ was used to study aerosol-derived airway morphometry (ADAM) in 79 healthy subjects, in order to establish reference values for a future clinical application of this technique. The reproducibility of the results over time was tested in two subjects over a period of 6 months. Since lung volumes of patients with lung disease are often altered, the influence of lung inflation on airspace dimensions was taken into account [11], and data were assessed at three levels of lung inflation.
Additionally, the relationship between airspace dimensions and anthropometric data, as well as parameters obtained from conventional lung function tests (spirometry and body plethysmography), were studied.

\section{Methods}

\section{Subjects}

Seventy nine subjects (38 males and 41 females) were included in the study. They were classified into four age groups in order to achieve an even age distribution: 20-29 yrs (20 subjects); 30-39 yrs (20 subjects); 40-49 yrs (20 subjects); and 50-60 yrs (19 subjects). Anamnestic data were collected using a questionnaire based on American Thoracic Society (ATS) recommendations [12]. Subjects were excluded from the study if they were active smokers or had smoked in the past more than 4 pack-years, or when there was any hint of respiratory or cardiovascular disease or atopy. Informed written consent was obtained from each subject. The protocol was approved by the Ethics Committee of the Medical School of the Ludwig-Maximilians University (Munich, Germany). 


\section{Aerosol-derived airway morphometry (ADAM)}

Monodisperse aerosol particles settle in calm air with a constant settling velocity (Vs). Within a porous medium, this gravitational motion leads to particle loss at inner surfaces, and the particle concentration is reduced with increasing time. Particle loss rate is high if the particles are located in a medium with small airspace dimensions, and particle loss rate is low in a medium with large dimensions. The decline in particle number concentration as a function of time can be used to calculate airspace dimensions [9].

This phenomenon can be used to non-invasively measure intrapulmonary airspace dimensions. Lungs were filled with aerosol particles of uniform number concentration by a tidal inspiration of a monodisperse aerosol. The inspired tidal volume can be considered to be composed of infinitesimally small volume elements, which penetrate to different volumetric lung depths $\left(V_{\mathrm{p}}\right)$. During a breathholding period $(t \mathrm{p})$, the particles settle onto airway surfaces, and the particle number concentration in each volume element decreases. This reduction of aerosol concentration in each volume element can be measured by assessing the particle-recovery ratio $(\mathrm{R})$ as a function of breathholding time (fig. 1). Recovery is defined as the ratio of particle number concentration in an exhaled volume element, and the concentration in the inhaled aerosol.

The relationship $\mathrm{R}=\mathrm{f}(t \mathrm{p})$ can be studied theoretically by considering either a model of randomly oriented identical tubes [13], or a chord-length model [2]. For both models:

$$
\mathrm{R}=\exp (-1.27 \mathrm{Vs} t \mathrm{p} / \mathrm{EAD})
$$

Effective airspace dimension (EAD) is calculated for each volumetric lung depth $V$ p by fitting an exponential function to the relationship between the various

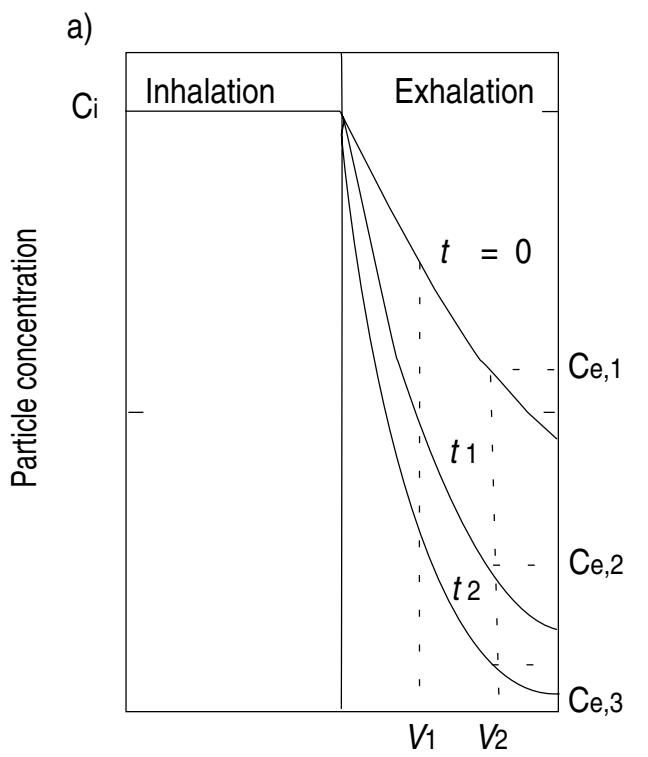

Respired volume recovery values, measured for each lung depth, $V_{\mathrm{p}}$, and the corresponding breath holding times $t \mathrm{p}$. For the tube model, EAD represents the tube diameter. In the proximal lung, EAD therefore represents the diameter of conducting airways. Since these airways at each lung depth are not identical tubes, EAD is called the effective airspace dimension. For the chordlength model, EAD characterizes the mean distance between the walls of a porous medium. In the peripheral lung, EAD is closely related to the mean linear intercept usually used to characterize peripheral lung geometry [2].

To compare EAD among subjects with different lung size, lung depth must be normalized:

$$
V \mathrm{p}, \mathrm{r}=V_{\mathrm{p}} / V_{\mathrm{L}}
$$

where $V \mathrm{~L}$ is the end-inspiratory lung volume at which the breathholding manoeuvre is performed [11].

\section{Instrumental set-up and inhalation protocol}

ADAM requires the measurement of the particle number concentration in the respired air as a function of the air volume. Both values, particle number concentration and air volume, are provided by the respiratory aerosol probe which is an on-line, open-flow system combining aerosol photometry and pneumotachography [14].

Subjects sat in front of the respiratory aerosol probe in an upright position. The extrathoracic dead space of the subject was reduced by a silicone dental compound, which filled part of the oral cavity and was connected to the mouthpiece of the respiratory aerosol probe.

After a few tidal breaths at functional residual capacity, the subject exhaled $50 \%$ of the expiratory reserve volume (ERV), as determined by body plethysmography. By use of computer controlled pneumatic air valves, the inspiration channel was then switched from supplying

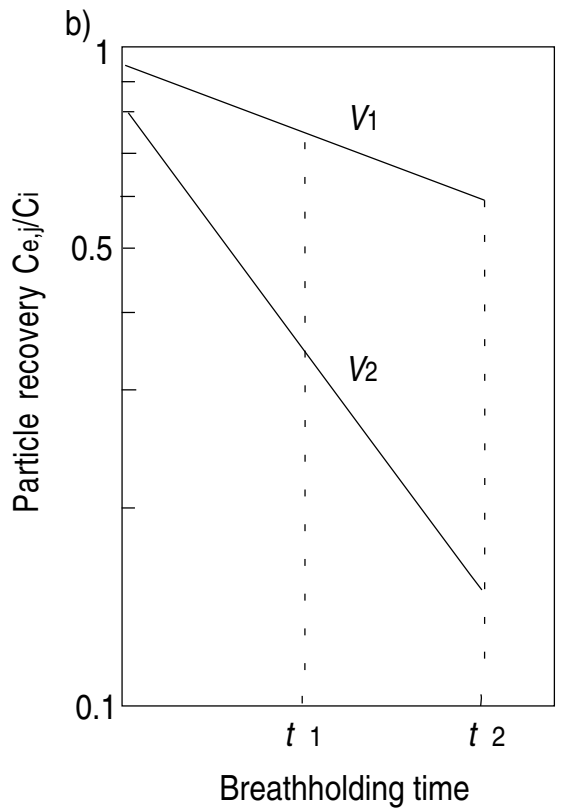

Fig. 1. - Principles of aerosol derived airway morphometry. a) Particle number concentration as a function of the respired volume; and b) aerosol recovery as a function of the breathholding time. $V_{\mathrm{j}}$ : different volumetric lung depths; Ce,k: particle number concentrations measured in a certain volumetric lung depth for different breathholding times $t \mathrm{k}$; Ci: average particle number concentration during inhalation. 
clean air to supplying aerosol, and the subject inhaled the aerosol volume:

$$
V \mathrm{a}=0.5 \mathrm{ERV}+\mathrm{f} \text { TLC }- \text { ITGV }
$$

at a constant airflow rate of $250 \mathrm{~mL} \cdot \mathrm{s}^{-1}$. The required flow rate was achieved by watching a visual airflow signal. $f$ is a fraction of total lung capacity (TLC), ITGV is the intrathoracic gas volume. For each subject, ADAM was assessed at three different levels of lung inflation, i.e. for end-inspiratory lung volumes of 50,70 or $85 \%$ TLC ( $\mathrm{f}=0.5,0.7$ or 0.85$)$. After a certain breathholding time $t \mathrm{p}$, the subject exhaled below functional residual capacity (FRC) with the same flow rate (fig. 2). This manoeuvre was performed for $t \mathrm{p}=2,4,6,8,10$, $12,14,16$ and $18 \mathrm{~s}$. Using the particle concentration data recorded at volume intervals of $0.5 \mathrm{~mL}$, aerosol recovery was calculated for the volumetric lung depths of $V_{\mathrm{p}}=100,150,200,250,300,400,500,600,700$ and $800 \mathrm{~mL}$, and the relative volumetric depths of $V_{\mathrm{p}, \mathrm{r}}=$ $0.02,0.04,0.06, \ldots, 0.30$. All volumes were corrected to body temperature, atmospheric pressure and saturation with water vapour (BTPS).

\section{Particle generation and classification}

Monodisperse di-2-ethylhexyl sebacate (DEHS) droplets suspended in nitrogen were produced by heterogeneous nucleation of DEHS vapour on $\mathrm{NaCl}$ nuclei, and then diluted with air. The terminal $\mathrm{V}_{s}$ of the droplets was measured in a convection-free sedimentation chamber. The average $V_{s}$ throughout this study was $23 \pm 2 \mu \mathrm{m} \cdot \mathrm{s}^{-1}$ corresponding to an average geometrical droplet diameter of $0.84 \mu \mathrm{m}$.

\section{Lung function test}

Each subject underwent conventional lung function tests before the ADAM measurements. Lung function was assessed in a constant volume body plethysmograph with integrated spirometer (Masterlab, Jäger, Würzburg,

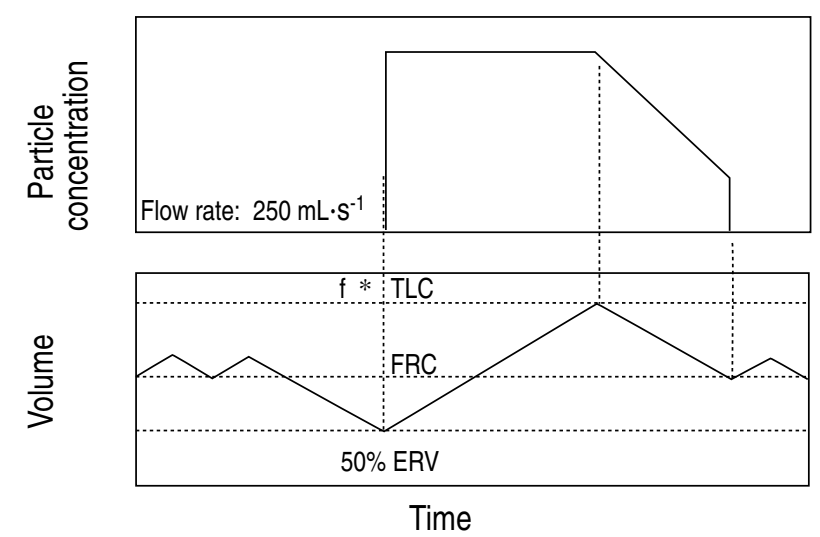

Fig. 2. - Schematics of the inhalation protocol. $f$ : a fraction of total lung capacity (TLC); FRC: functional residual capacity; ERV: expiratory reserve volume.
FRG) $[15,16]$. Three maximal forced expirations were recorded, and the flow-volume curve which yielded the highest sum of forced expiratory volume in one second (FEV1) and vital capacity (VC) was selected for analysis. The following parameters of interest were included in the data analysis: specific airway resistance (sRaw), TLC, residual volume (RV), ITGV, VC, ERV, peak expiratory flow (PEF), FEV1, and the maximal expiratory flows at 25, 50 and 75\% vital capacity (MEF25, MEF50, MEF75). Predicted values for conventional lung function parameters were calculated by normalization to the reference values proposed by the European Coal and Steel Community [15].

\section{Data analysis}

In order to compare results of ADAM with morphometric data from the lung, the symmetrical lung models described by Weibel [17] and Yeh and Schumm [18] were scaled isotropically to $70 \%$ TLC and the volume of the instrumental dead space $(20 \mathrm{~mL})$ was added.

For each volumetric lung depth the intrasubject variability of EAD was quantified by calculating the relative standard deviation, s (standard deviation divided by the mean of the repeated EAD measurements from one subject for each lung depth). Intersubject variability was quantified by the standard deviation of EAD measured in all subjects divided by the mean value of EAD (relative population standard deviation, $\sigma$ ).

To assess correlations between EAD and anthropometric parameters or parameters of conventional lung function tests, it must be kept in mind that such correlations are possibly masked by partial correlations among these parameters. In order to take such partial correlations into consideration, a linear multiple regression was calculated. The following linear model was assumed: $\mathrm{EADX}=\mathrm{f}$ (gender, age, height, weight, TLC, RV, VC, ERV, FEV1, MEF25, MEF50, MEF75, PEF, sRaw). The influence of each of these parameters on EAD was adjusted for the influence of any other parameter. Multiple regression analysis was performed using the SASsoftware package on an IBM 4381 computer (operating system CMS). Results were considered to be statistically significant at error levels of $\mathrm{p}$ less than 0.01 .

\section{Results}

Table 1 summarizes the anthropometric and lung function parameters of the study population. Table 2 shows the median, 5th percentile, and 95th percentile of EAD measured for each relative lung depth. EAD as a function of relative volumetric lung depth measured at $70 \%$ TLC is illustrated in figure 3. For shallow lung depths $(V \mathrm{p}, \mathrm{r}=0.02)$ the mean EAD is about $3 \mathrm{~mm}$. With increasing lung depth, EAD decreases rapidly and then, beyond $V_{\mathrm{p}, \mathrm{r}}=0.10$, EAD remains nearly constant at about 0.27 $\mathrm{mm}$. The morphometric data of WeIBEL [17] and YEH and Schumm [18] show reasonable agreement with these experimental data. However, in shallow lung depths, the measured EAD to $V_{\mathrm{p}, \mathrm{r}}$ relationship is less steep than expected from the lung models. In the lung periphery, 
Table 1. - Anthropometric and lung function parameters of the study population

\begin{tabular}{|c|c|c|}
\hline Parameter & Mean & $\%$ pred \\
\hline Age yrs & $39 \pm 11$ & \\
\hline Height $\mathrm{m}$ & $1.72 \pm 0.09$ & \\
\hline Weight $\mathrm{kg}$ & $68 \pm 11$ & \\
\hline TLC $\quad$ L & $6.3 \pm 0.09$ & 103 \\
\hline RV & $1.6 \pm 0.4$ & 88 \\
\hline $\mathrm{VC}$ & $4.6 \pm 1$ & 109 \\
\hline FEV1 & $3.7 \pm 0.8$ & 108 \\
\hline MEF25 L·s $\mathrm{s}^{-1}$ & $1.7 \pm 0.6$ & 89 \\
\hline MEF50 $\mathrm{L} \cdot \mathrm{s}^{-1}$ & $4.6 \pm 1.3$ & 100 \\
\hline MEF75 L·s ${ }^{-1}$ & $7.7 \pm 1.8$ & 110 \\
\hline $\mathrm{PEF} \quad \mathrm{L} \cdot \mathrm{s}^{-1}$ & $9.6 \pm 2.7$ & 117 \\
\hline $\mathrm{kPa} \cdot \mathrm{s}^{-1}$ & $0.59 \pm 0.14$ & \\
\hline
\end{tabular}

Relative values of lung function parameters are given as percentage of the predicted value (\% pred), [15]. Values are presented as mean \pm SD. TLC: total lung capacity; RV: residual volume; VC: vital capacity; FEV1: forced expiratory volume in one second; MEF25, MEF50 and MEF75: maximal expiratory flow at 25, 50 and $75 \% \mathrm{VC}$, respectively; PEF: peak expiratory flow; sRaw: specific airway resistance.

Table 2. - Effective airspace dimension (EAD) as a function of relative volumetric lung depth $\left(V_{p, r}\right)$ measured in 79 healthy subjects at different levels of lung inflation, expressed as percentage of total lung capacity (TLC)

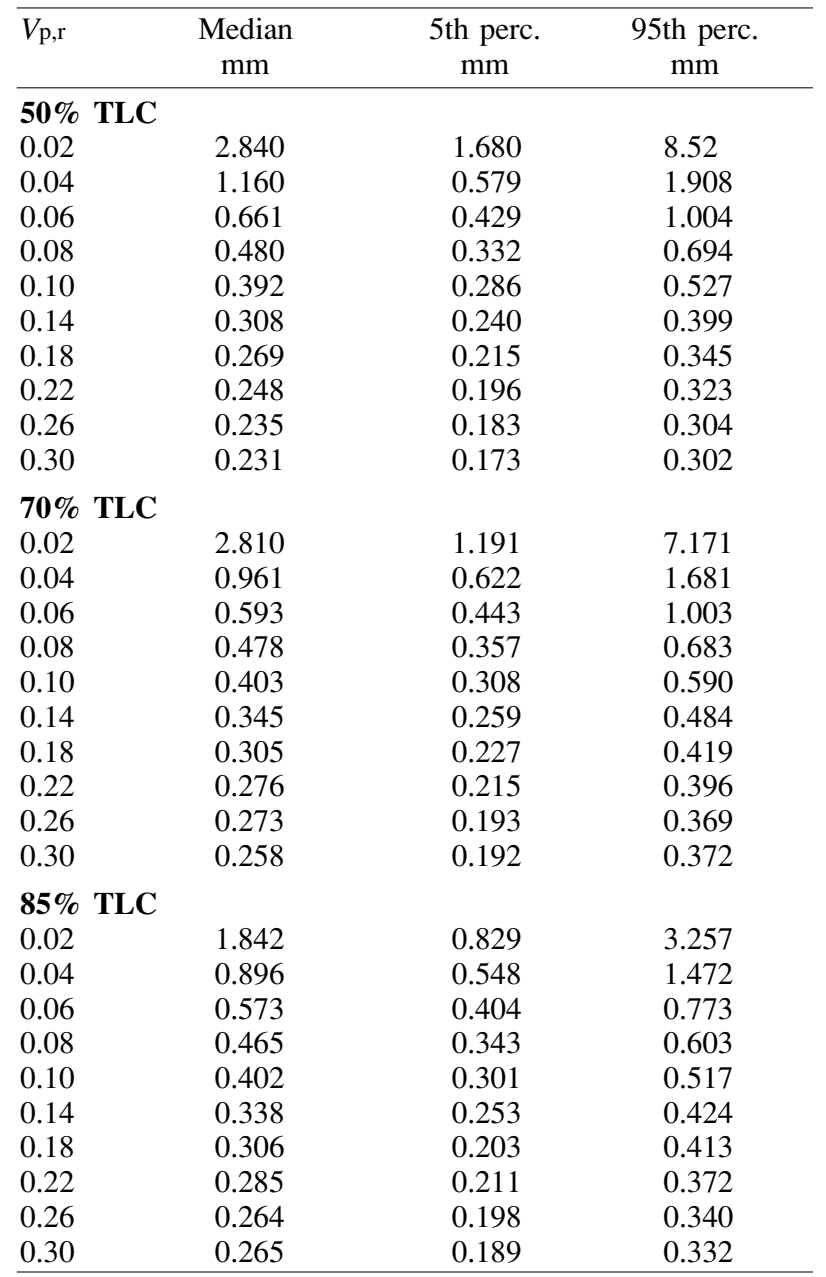

Values are presented as median, and 5th and 95th percentile (perc.).

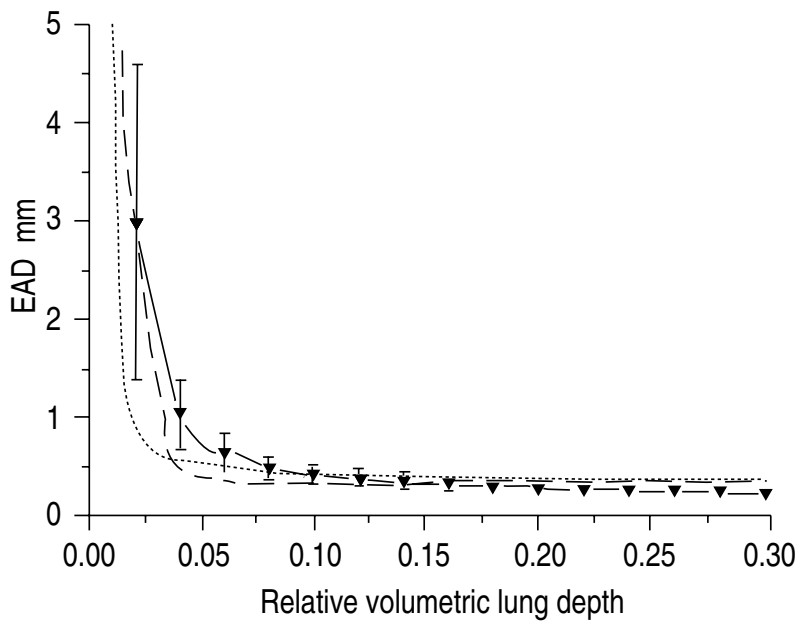

Fig. 3. - Effective airspace dimensions (EAD) (mean \pm SD) as a function of relative volumetric lung depth measured in 79 healthy subjects compared to morphometric lung models. $\boldsymbol{\nabla}$ : aerosol-derived airway morphometry (ADAM); …..... WeIBEL [17] model A; - - - : YeH and SCHumm [18].

for volumetric lung depths beyond $V \mathrm{p}, \mathrm{r}=0.1$, EAD is somewhat smaller than the morphometrically assessed airspace size.

In order to assess the variability of ADAM over time, EAD was measured three times a month over a period of 6 months for two of the subjects. Table 3 shows the relative standard deviation, $\mathrm{s}$, over all 18 measurements of EAD obtained from both subjects at each volumetric lung depth. Relative standard deviation is highest in shallow lung depths and decreases with increasing lung depth. For relative volumetric lung depths beyond $V_{\mathrm{p}, \mathrm{r}}$ $=0.2$, relative standard deviation again increases. Except for very shallow lung depths, variability of EAD was in the order of $10 \%$.

Figure 4 shows mean EAD obtained at three different levels of lung inflation. Figure 4a shows EAD as a function of the absolute volumetric lung depth, $V \mathrm{p}$; and figure $4 \mathrm{~b}$ shows EAD as a function of the relative volumetric lung depth, $V$ p,r. EAD as a function of $V$ p shows a much higher dependency on lung inflation than EAD as a function of $V$ p,r. EAD measured at $85 \%$ TLC is in all

Table 3. - Relative standard deviation of effective airspace dimension (EAD) measured in two subjects (Nos. 1 and 2) three times a month over a period of 6 months (18 single measurements), as a function of relative volumetric lung depth $V_{p, r}$

\begin{tabular}{ccc}
\hline$V_{\mathrm{p}, \mathrm{r}}$ & $\begin{array}{c}\text { No. } 1 \\
\%\end{array}$ & $\begin{array}{c}\text { No. } 2 \\
\%\end{array}$ \\
\hline 0.02 & 18 & 23 \\
0.04 & 14 & 11 \\
0.06 & 14 & 7 \\
0.08 & 14 & 6 \\
0.10 & 11 & 6 \\
0.14 & 10 & 6 \\
0.18 & 9 & 6 \\
0.22 & 9 & 8 \\
0.26 & 12 & 8 \\
0.30 & 14 & 11 \\
\hline
\end{tabular}


a)

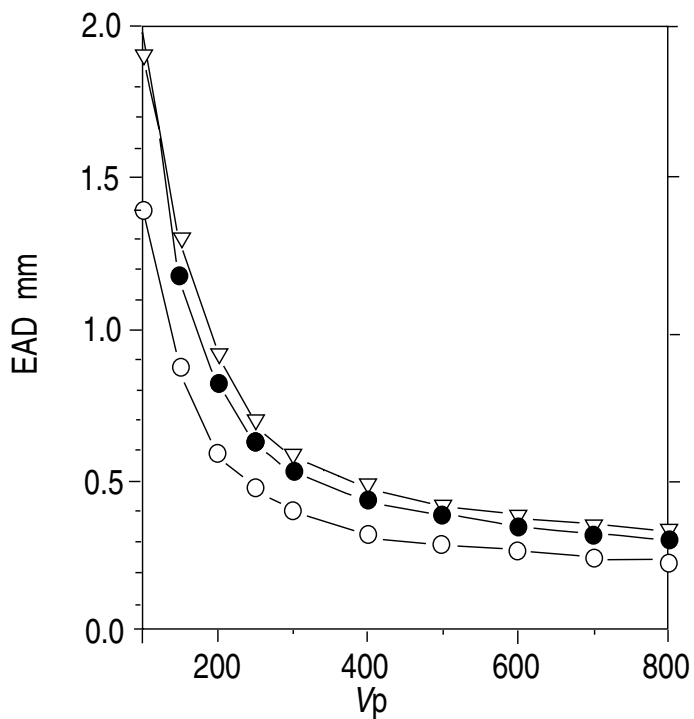

b)

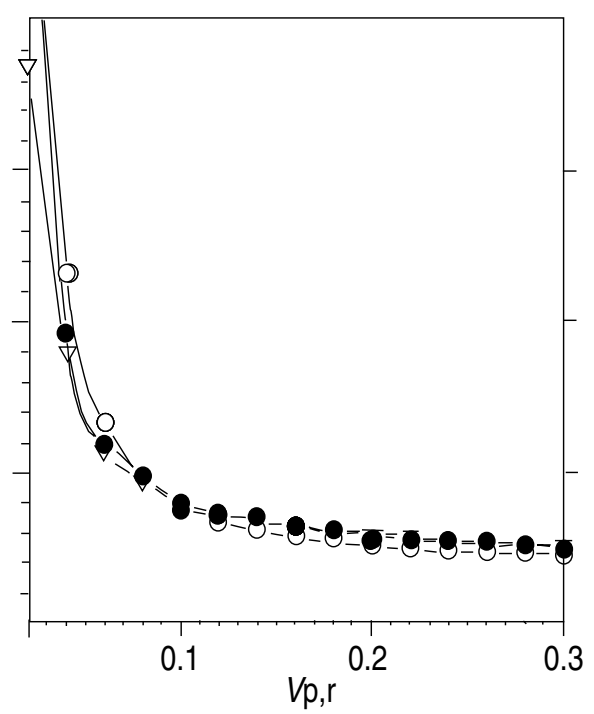

Fig. 4. - Effective airspace dimensions (EAD) measured at three different levels of lung inflation as a function of: a) absolute volumetric lung depth $\left(V_{\mathrm{p}}\right)$ : and b) relative volumetric lung depth. $\left(V_{\mathrm{p}, \mathrm{r})}\right.$. - $-: 50 \%$ total lung capacity (TLC); - $: 70 \%$ TLC; $-\nabla-: 85 \%$ TLC.

a)

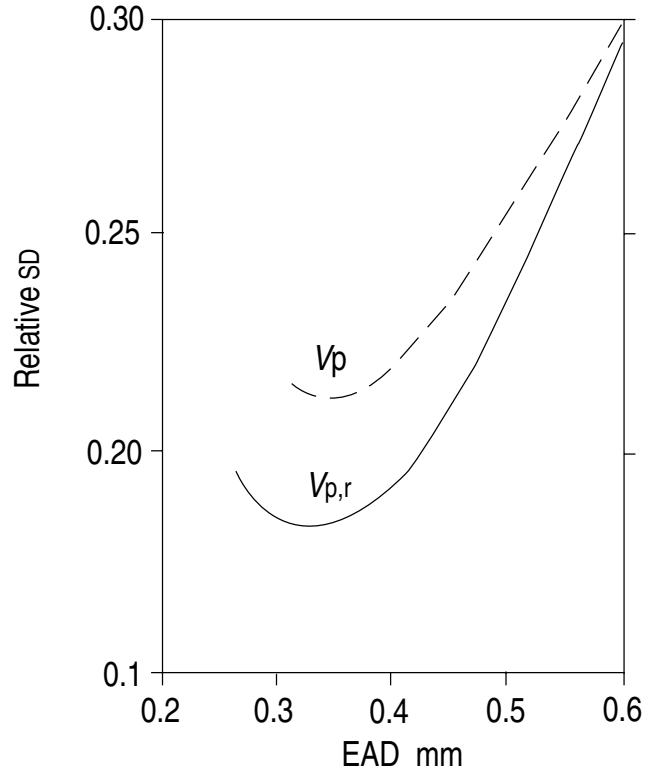

b)

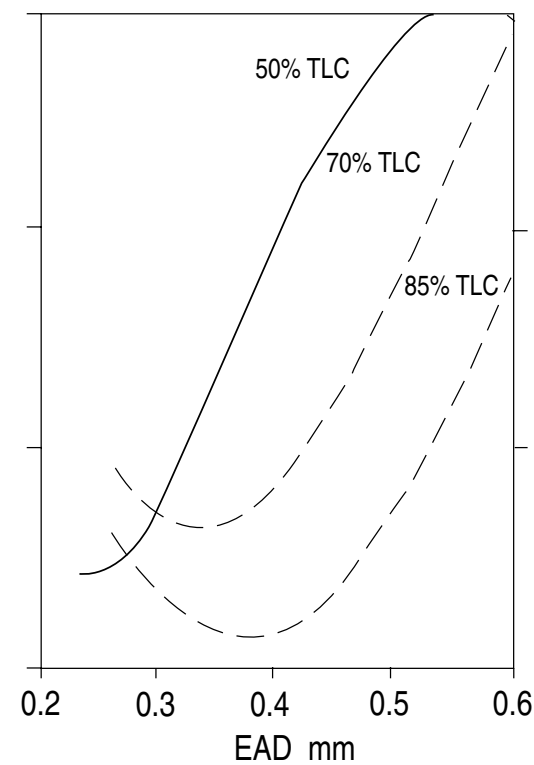

Fig. 5. - Relative population standard deviation of effective airspace dimensions (EAD) as a function of the average EAD measured for: a) absolute volumetric lung depth $\left(V_{\mathrm{p}}\right)$ and relative volumetric lung depth $\left(V_{\mathrm{p}, \mathrm{r}}\right)$; and $\left.\mathrm{b}\right)$ for three levels of lung inflation, expressed as percentage of total lung capacity (TLC).

absolute lung depths on average $46 \%$ higher than the value measured at $50 \%$ TLC. In relative volumetric lung depths beyond $V_{\mathrm{p}, \mathrm{r}}=0.1$ the difference between EAD measured at 50 and $85 \%$ TLC is about $15 \%$, which is in agreement with the model of isotropic lung inflation [19].

Variability of EAD can be reduced by normalizing lung depth to end-inspiratory lung volume $(V \mathrm{~L})$. Figure 5 shows the relative population standard deviation, $\sigma$, as a function of EAD. Figure 5a shows that $\sigma$ decreases with decreasing airspace size. Furthermore, it can be seen that $\sigma$ measured in relative volumetric lung depths is $20-30 \%$ smaller than $\sigma$ measured in absolute lung depths. Figure $5 \mathrm{~b}$ illustrates the dependence of $\sigma$ on lung inflation. $\sigma$ measured at $85 \%$ TLC is as much as $60 \%$ smaller than $\sigma$ measured at $50 \%$ TLC.

For relative volumetric lung depths, multiple regression analysis shows a significant $(\mathrm{p}<0.01)$ dependency of EAD on the subject's age (table 4). For lung inflation $50 \%$ TLC this dependency was significant in relative volumetric lung depths $V_{\mathrm{p}, \mathrm{r}}>0.08$, for $70 \%$ TLC in depths $V_{\mathrm{p}, \mathrm{r}}>0.06$, and for $85 \%$ TLC even in lung depths $V_{\mathrm{p}, \mathrm{r}}$ $>0.04$. No other significant dependencies were observed. 
Table 4. - Significant correlations between effective airspace dimension (EAD) measured in various relative volumetric lung depth $\left(V_{p, r}\right)$ at different levels of lung inflation, expressed as percentage of total lung capacity (TLC), and anthropometric and conventional lung function parameters estimated by multiple regression

\begin{tabular}{lcl}
\hline 50\% TLC & \multicolumn{1}{c}{ parameter } \\
\hline & 0.02 & \\
0.04 & \\
0.06 & \\
0.08 & Age $(0.0006)$ \\
& 0.10 & Age $(0.0006)$ \\
$>0.10$ & Age $(0.0002)$ \\
& & \\
$\mathbf{7 0 \%}$ TLC & 0.02 & \\
& 0.04 & \\
& 0.06 & Age $(0.0001)$ \\
& 0.08 & Age $(0.0001)$ \\
& 0.10 & Age $(0.0004)$ \\
$>0.10$ & Age $(0.0004)$ \\
& \\
& 0.02 & \\
& 0.04 & Age $(0.005)$ \\
& 0.06 & Age $(0.001)$ \\
& 0.08 & Age $(0.0006)$ \\
& 0.10 & Age $(0.003)$ \\
& $>0.10$ & Age $(0.01)$ \\
\hline
\end{tabular}

The significance level of the relationship is given in parenthesis.

\section{Discussion}

\section{ADAM versus lung models}

The characterization of intrapulmonary airspace dimensions using aerosol-derived airway morphometry is in reasonable agreement with the symmetrical morphometric Weibel model A [17] and the YeH and Schumm model [18]. However, some differences are evident. Both lung models predict considerably larger airspace dimensions in relative volumetric lung depths beyond $V \mathrm{p}, \mathrm{r}>0.15$ than those measured using the aerosol technique. For these lung depths, the Weibel model predicts an airspace calibre of $0.4 \mathrm{~mm}$, the Yeh and Schumm model predicts $0.38 \mathrm{~mm}$; whereas, ADAM measured an EAD of $0.26 \mathrm{~mm}$. This discrepancy might be due to the fact that both lung models approximate the lung by a simple serial structure in which all alveoli are located beyond the 23rd bronchial generation. In such a model, alveoli are located in a volumetric lung depth of 1,100 $\mathrm{mL}$ (Yeh and Schumm) or 1,500 $\mathrm{mL}$ (Weibel). In the real lung, on the other hand, the same anatomical structure is located in different airway generations. Alveoli may be reached from respiratory bronchioles (17th-19th generation) as well as from alveolar ducts (20th-22nd generation). The path-length to reach alveolar structures, which have an inner diameter of about $0.25 \mathrm{~mm}$ [20], therefore differs considerably throughout the lung. Alveoli might, therefore, contribute considerably to EAD measured in volumetric lung depths $0.15<V_{\mathrm{p}, \mathrm{r}}<0.3$. This may explain why EAD values are smaller than airspace dimensions predicted by the symmetrical lung models.

For relative volumetric lung depths between about 0.06 and 0.1 , airspace dimensions measured with the aerosol technique are higher than those predicted by the models. The relationship between airspace dimensions and lung depth given by the lung models exhibits in this region (generation 19-21) a much higher bend than the relationship measured using ADAM. This difference may, likewise, be explained by the fact that ADAM measures the average diameter of airways located at different airway generations, and this causes a smoothing of the steep bend predicted by the models. The smoothing effect on the measured EAD curve is amplified by the resolution power of this technique. It has recently been shown that the longitudinal resolution power of ADAM is decreased by the mechanisms of convective gas mixing [4]. The resolution power decreases with increasing lung depth, and this results in longitudinal averaging of airspace diameters.

\section{Effect of lung inflation}

As reported previously [11], the measurement of airspace dimensions with ADAM is hampered by the volumetric definition of the measuring site. When the lung volume is increased by inflating the lung, a certain absolute volumetric lung depth represents different anatomical sites within the lung; when the lung volume is increased, the measurement site as defined by $V_{\mathrm{p}}$ is shifted proximally and vice versa. Increasing the level of lung inflation results in a real increase of airspace calibre, and in an apparent increase due to the proximal shift of the measuring site. The results of this study support the approach of using a relative volumetric lung depth to overcome this artefact. For $V \mathrm{p}, \mathrm{r}>0.1$, dependency of EAD on the level of lung inflation is reduced to a degree which is of the same order as that expected from the model of isotropic lung inflation [19]. However, for more proximal airways the effect of the proximal shift of the measuring site is overcompensated by normalizing $V_{\mathrm{p}}$ to the lung volume. This results in a slight decrease of proximal EAD with increasing lung inflation. Presumably, this effect is due to a lower distensibility of proximal airways [21].

\section{Variability of EAD}

The introduction of relative volumetric lung depths notably reduces the population standard deviation of EAD. This decrease in variability is presumably due to the reduction of differences in EAD among subjects with different lung volume, which were observed in absolute volumetric lung depths. Relative volumetric lung depths appear to be more suitable than absolute volumetric lung depths for comparing EAD among subjects.

In addition, variability of EAD can be used to characterize basic features of ADAM. The variability of airspace dimensions, between individuals as well as within 
an individual, decreases with increasing lung depth. For airways with an EAD of about $2 \mathrm{~mm}$, the population standard deviation is more than three fold higher than that observed for peripheral airways. There are two explanations for this observation. Since EAD $\sim 1 / \log (\mathrm{R})$, the accuracy of the EAD measurement decreases rapidly with increasing aerosol recovery from the lung. But the variability of EAD is even more affected by the uncertainty in volumetric lung depth. This lung depth is defined as the volume between the sensitive volume of the aerosol photometer and those lung structures within which a certain aerosol volume element is located during the breathholding period. It includes the oral cavity and the pharynx. Both volume fractions are quiet variable. RivLin et al. [22] measured oropharyngeal volumes in healthy male subjects and found a $20 \mathrm{~cm}^{3}$ intersubject variation, which will cause a corresponding variation in volumetric depth. Since the relationship between EAD and the volumetric lung depth is very steep for $V_{\mathrm{p}, \mathrm{r}}$ $<0.05$, this variation in volumetric depth results in a considerable variation in EAD, and may be assumed to be responsible for the high intersubject variability observed in those proximal lung depths. In more peripheral airways, this uncertainty in volumetric lung depth is of little importance, since EAD is nearly independent of lung depth.

This result is important for future clinical applications of the technique: ADAM seems to allow the estimation of peripheral airspace dimensions with high accuracy and reproducibility. This capability is especially interesting since most common lung function tests (spirometry, body plethysmography) are not sensitive enough to detect early changes in peripheral lung geometry [23]. On the other hand, the measurement of proximal EAD using ADAM is less reproducible and less accurate. However, since conventional lung function tests are powerful tools to detect changes in calibre in proximal airways, there is no need for new diagnostic techniques to detect changes in such lung regions. Thus, ADAM appears to be appropriate for the detection of structural changes in peripheral airways and would be best applied as a supplement to conventional lung function tests.

Furthermore, intersubject variability is dependent on lung inflation; with increasing lung inflation the population standard deviation decreases. The difference between the population standard deviation measured at 50 and $85 \%$ TLC reaches $50 \%$. This result indicates that the application of ADAM should be performed at high levels of lung inflation, where the lungs of different subjects are more similar than at low lung volumes.

Correlation with age, anthropometric data, and conventional parameters

The potential use of ADAM as a diagnostic tool becomes evident after analysis of correlations of EAD with other lung function parameters or anthropometric data. Peripheral EAD are only dependent on the age of the subject; peripheral EADs increase with increasing age. This phenomenon is well-known from the literature [24, 25],

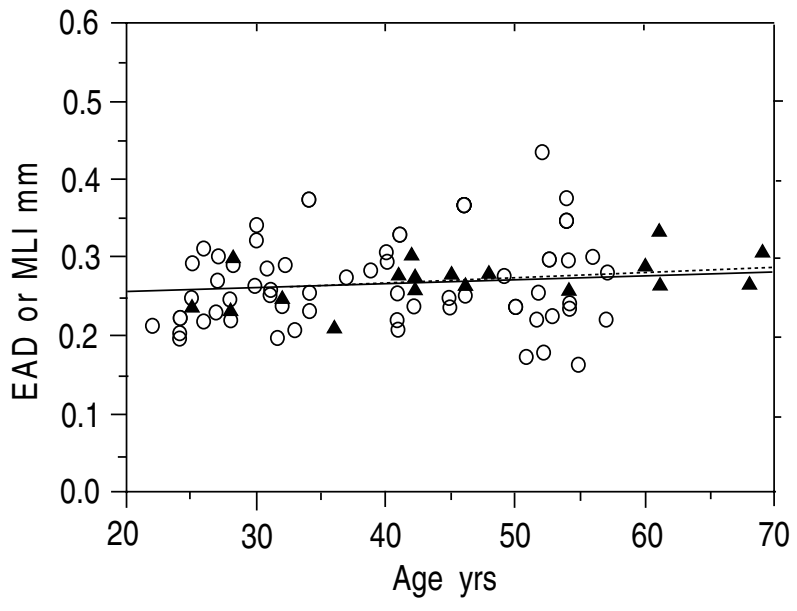

Fig. 6. - Relationship between the age of a subject and effective airspace dimensions (EAD) measured in a relative volumetric lung depth $\left(V_{\mathrm{p}, \mathrm{r}}\right)=0.3$ compared to mean linear intercepts (MLI) measured by THuRLBECK [24]. -○- : aerosol-derived airspace morphometry (ADAM); $\cdot \boldsymbol{\Delta} \cdot \cdot \cdot$ : ThURLBECK [24].

and is explained by a loss of elasticity in ageing lungs. Figure 6 shows EAD at lung depth $V \mathrm{p}, \mathrm{r}=0.3$ as a function of the subject's age. Also shown in this figure is the relationship between the mean linear intercept (MLI) and age measured in autopsy lungs [24]. The observed excellent agreement between morphometrical and aerosol-derived data, and between the slopes of the relationships EAD versus age and MLI versus age, further support the hypothesis that ADAM is a sensitive tool for detecting small changes in peripheral lung geometry.

In conclusion, in this study, aerosol-derived airway morphometry was used to non-invasively study airspace dimensions in 79 healthy subjects, in order to assess reference data for the future clinical application of ADAM and to investigate the effect of lung inflation, anthropometric, and lung function parameters on ADAM. The results show that peripheral airspace dimensions are in good agreement with anatomical data. The absolute sizes, the distension behaviour, and the age dependency of peripheral airspace calibre were observed to be similar to results described in the anatomical literature. For peripheral airspaces a very low intersubject variability was observed when EAD was expressed as a function of relative volumetric lung depth and the measurement was performed at a high level of lung inflation. Taking advantage of this small variability should lead to improved detection of small changes in peripheral airway geometry.

\section{References}

1. Nikiforov AI, Lippmann M, Palmes FD. Validation of an in vivo aerosol probe technique by measurements of deposition and morphometry in excised human lungs. Ann Occup Hyg 1988; 32: 33-39.

2. Rosenthal FS. Aerosol recovery following breatholding derived from the distribution of chordlength in pulmonary tissue. J Aerosol Sci 1989; 20: 267-277.

3. Rosenthal FS, Wright S. Assessment of papain-induced 
lung injury in isolated lungs by measurements of aerosol deposition and mixing. J Appl Physiol 1992; 72: 459-467.

4. Brand P, Selzer T, Tuch T, Schulz A, Heyder J. Accuracy and resolution power of aerosol derived airway morphometry in a simple lung model. Exp Lung Res 1994; 20: $185-205$.

5. Hankinson JL, Palmes ED, Lapp NL. Pulmonary airspace size in coal-miners. Am Rev Respir Dis 1979; 11 9: 391-397.

6. Palmes ED, Goldring RM, Wang CS, Altshuler B. Effect of chronic obstructive pulmonary disease on rate of deposition of aerosols in the lung during breathholding. In: Walton WH, ed. Inhaled Particles III. Old Woking Surrey, Union Brothers Ltd, 1971.

7. Bennett WD, Smaldone GC. Use of aerosols to estimate mean airspace size in chronic obstructive pulmonary disease. J Appl Physiol 1988; 64: 1554-1560.

8. Beinert T, Brand $\mathrm{P}, \mathrm{Behr} \mathrm{J}$, et al. Aerosol-derived intrapulmonary airspace dimensions in emphysematic patients. Eur Respir J 1990; 10: 242.

9. Heyder J. Assessment of airway geometry with inert aerosols. J Aerosol Med 1989; 2: 89-97.

10. Blanchard JD, Heyder J, O'Donnell CR, Brain JD. Aerosolderived lung morphometry: comparisons with a lung model and lung function indices. J Appl Physiol 1991; 71: 1216-1224.

11. Brand P, Beinert T, Hillebrecht A, Heyder J. Aerosolderived airway morphometry at different levels of lung inflation. J Aerosol Med 1993; 6: 165-176.

12. Ferris BG. Recommended respiratory disease questionaires for use with adults and children in epidemiological research. Am Rev Respir Dis 1978; 118: 7-55

13. Heyder J. Gravitational deposition of aerosol particles within a system of randomly orientated tubes. Aerosol Sci 1975; 6: 133-137.
14. Gebhart J, Heigwer G, Heyder J, Roth C, Stahlhofen W. The use of light scattering photometry in aerosol medicine. J Aerosol Med 1988; 1: 89-112.

15. Quanjer PH, Tammeling GJ, Cotes JE, Pedersen OF, Peslin R, Yernault JC. Lung volumes and forced ventilatory flows. Eur Respir J 1993; 6 (Suppl. 16): 5-40.

16. DuBois AB, Botelho SY, Comroe JH. A new method for measuring airway resistance in man using a body plethysmograph. J Clin Invest 1956; 35: 327.

17. Weibel ER. Morphometry of the human lung. Berlin, Springer-Verlag, 1963.

18. Yeh HC, Schumm GM. Models of human lung airways and their application to inhaled particle deposition. Bull Math Biol 1980; 42: 461-480.

19. Hughes JMB, Hoppin FG, Mead J. Effect of lung inflation on bronchial length and diameter in excised lungs. J Appl Physiol 1972; 32: 25-35.

20. Haefeli-Bleuer B, Weibel E. Morphometry of the human pulmonary acinus. Anat Rec 1988; 220: 401-414.

21. Tisi GM, Minh VD, Friedman PJ. In vivo dimensional response of airways of different size to transpulmonary pressure. J Appl Physiol 1975; 39: 23-29.

22. Rivlin J, Hoffstein V, Kalbfleisch J, McNicholas W, Zamel N, Bryan C. Upper airway morphology in patients with idiopathic obstructive sleep apnea. Am Rev Respir Dis 1984; 129: 355-360.

23. Wright JL, Cagle P, Churg A, Colby TV, Myers J. Diseases of the small airways. Am Rev Respir Dis 1992; 146: $240-262$.

24. Thurlbeck WM. The internal surface area of nonemphysematic lungs. Am Rev Respir Dis 1967; 95: 765773.

25. Niewoehner DE, Kleinerman J, Liotta L. Elastic behavior of postmortem human lungs: effects of ageing and mild emphysema. J Appl Physiol 1975, 39: 943-949. 